\section{NP30 (continued)}

for low-literacy Spanish speakers. Validation will be done by comparing MCMT item responses to behaviors observed during mealtime interactions with the target child (final target $\mathrm{N}=80$ ). Content of the tool and mealtime coding system was based on previous validation results of the English MCMT version. The process described here will be used to assess whether parent responses accurately capture their mealtime behaviors.

Evaluation: Videotaping of mealtimes is underway (current $n=22$ ). Coder reliability has been established and final coding is complete for one quarter of the collected videos. Approximately 35 videos will be finalized by the time of the report. Development of the coding system and preliminary descriptive data of the observed parent mealtime behaviors will be reported. Preliminary associations between behaviors and MCMT responses will be shared.

Conclusion and Implications: Development of a valid Spanish language self-assessment tool with low-literacy demands to capture feeding style would be an effective tool for federally funded nutrition education programs. Such tools enable programs to tailor their messaging to key barriers specific to each group.

Funding: 2015-68001-23280

\section{NP31 Spanish Adaptation of a Pictorial Assessment of Diet Quality}

Karina Diaz Rios, PhD, University of California, Merced; Gloria Zavala, BS, University of California, Davis; Maria Espinoza, BS; Nancy Keim, PhD, Western Human Nutrition Research Center, USDA; Mical Kay Shilts, PhD, California State University, Sacramento;

Marilyn Townsend, PhD, RD, mstownsend@ucdavis.edu, University of California, Davis, 1 Shields Avenue, Davis, CA 95616

Objective: To describe the process of validating a short pictorial diet quality assessment tool to be used with lowincome Spanish-speakers.

Description: Latinos are disproportionally affected by nutrition-related conditions. A proportion of Spanish speakers in the US have limited ability to speak English. Linguistically and culturally appropriate evaluation tools are necessary to obtain valid and reliable dietary data from this population. Previous research has shown vegetable variety to be a proxy for diet quality and can serve as an alternative to the more burdensome 24-hour diet recall. A Spanish version of the tool was developed simultaneously with an English version. Bilingual and native Spanish speakers are involved in a multistage, iterative process that include face-validation through cognitive interviews, forward translation, and examination of conceptual consistency by subject-matter experts.

Evaluation: Text and pictures in the tool are examined for clarity, understanding, and relevance through cogni- tive interviews with a convenience sample of Spanishspeaking parents of 2-5 year old children at three Head Start sites in northern California. The tool is modified after interviewees' feedback and content fidelity appraisal by experts in the research team. The process is iterated until satisfaction is achieved and no further changes are desirable.

Conclusions and Implications: Linguistic and cultural considerations are part of the process of achieving content and face validity of a short pictorial diet quality assessment tool to be used with low-income Spanishspeakers.

Funding: 2015-68001-23280

\section{NP32 Smarter Lunchrooms Randomized Control Trial: Results from Year 4}

Katherine Greene, MPH, kng29@cornell.edu, Cornell University, 475 Warren Hall, Ithaca, NY 14850; Gnel Gabrielyan, PhD, Cornell University; Adam Brumberg; Jamie Dollahite, PhD; David R. Just, PhD; Brian Wansink, PhD

Objective: In year four, the research objective was to test the impact of implementing fewer Smarter Lunchrooms protocol items, and food service ownership of Smarter Lunchrooms intervention, on the selection and consumption of fruits, vegetables, and while milk in middle schools. This objective was based on previous process evaluation findings of varying implementation fidelity.

Description: In partnership with Cornell Cooperative Extension, 18 middle schools from urban districts in Upstate New York participated in the project during the spring 2016 semester. Schools were allocated into three groups: selection, matched/ assigned, and control. Selection schools created their own intervention from a list of protocols; matched/ assigned schools were matched with selection school based on practices, and assigned same protocols; and control schools made no changes to existing set up. The schools selected or were assigned two Smarter Lunchroom protocols from each category (fruits, vegetables, white milk), for a total of six protocols. Each protocol was targeted at increasing the convenience, visibility, and attractiveness of fruits, vegetables, and white milk.

Evaluation: Selection, waste, and consumption of food items were measured by trained researchers using the quarter-waste method of visual estimation to determine tray waste. Results were compared between treatment and control schools.

Conclusions and Implications: Preliminary results show allowing schools to choose their own protocols had a greater impact on the selection and consumption of fruits, compared to the schools who were told what protocols to implement. Further analyses are ongoing to compare selection schools with their matched counterparts, along with the inclusion of process data. This will

Continued on page $S 123$ 
NP32 (continued)

allow us to evaluate the impact of implementation fidelity on the intervention results.

Funding: 2012-68001-19604

\section{NP33 Process Evaluation of the Smarter Lunchrooms Randomized Controlled Trial (RCT): Year 3}

Alisha Gaines, PhD, againes@cornell.edu, Cornell University, 342A MVR Hall, Reservoir Avenue, Ithaca, NY 14853; Tisa Hill, MPH, Cornell University;

Jamie Dollahite, $\mathrm{PhD}$

Objective: The purpose of this study was to conduct process evaluation of the Smarter Lunchrooms RCT, redesigned to examine effectiveness of environmental strategies promoting fruit, vegetable, and unsweetened milk consumption in New York State middle schools that self-selected intervention components, compared with schools that were assigned an intervention protocol.

Description: Participating schools were randomized to receive no intervention (control schools, $n=4$ ), a six-week intervention in which food service staff selected protocol items (self-selection schools, $n=3$ ), or an assigned protocol mirroring that of a self-selection school with similar sociodemographic characteristics (matched schools, $n=5$ ). The research team partnered with Cornell Cooperative Extension (CCE) agents to deliver the intervention. An adapted RE-AIM framework guided process evaluation designed to monitor fidelity to intervention protocols, determine the extent to which protocols were maintained post-intervention, and identify facilitators and barriers to successful implementation and maintenance.

Evaluation: Measures included training documentation, school nutrition environment assessments, cafeteria audits, CCE school contact logs, and post-intervention interviews with CCE and food service staff. Implementation fidelity and maintenance were higher than in previous iterations of this RCT, though no consistent differences were observed among self-selection and matched schools. Non-compliance was associated with limitations in school food offerings, restrictive serving line structures, and changes to staff routines that proved difficult to maintain in busy serving periods. Staff motivation and effective intervention support facilitated successful implementation.

Conclusions and Implications: Leveraging staff motivation and providing support for managing barriers to implementation proved effective in enhancing implementation fidelity. These data will inform analyses of intervention outcomes and may prove valuable for other environmentally-focused interventions in school cafeterias.

Funding: 2012-68001-19604

\section{NP34 Outputs and Outcomes at Year 5 of the Out-of-School Program for Youth and Adult Dyads: iCook 4-H}

Adrienne White, PhD, RDN, FAND, awhite@maine.edu, University of Maine, 5735 Hitchner Hall, Orono, ME
04469-5735; Lisa Franzen-Castle, PhD, RN, University of Nebraska-Lincoln; Kendra Kattelmann, PhD, RDN, South Dakota State University; Sarah Colby, PhD, RD, University of Tennessee; Melissa Olfert, DPH, RDN, West Virginia University

Objective: To present primary outputs and outcomes at year 5 of iCook $4-\mathrm{H}$

Description: iCook 4-H was a 2 -year control/treatment intervention study ( $\mathrm{n}=228$ dyads) followed by dissemination testing ( $n=57$ dyads). The final curriculum is 8,2 hour sessions designed to impact health-promoting behavior for 9-10 year olds and their adult food preparer. The content includes food preparation and family activities to encourage cooking, eating, and playing together. Four seasonal newsletters serve as boosters following sessions. Evidence-based pre/post program outcome evaluations of dyads with a fidelity instrument to test intended versus actual implementation accompany the curriculum. A curriculum implementation training course is on the eXtension Campus Moodle website and in development is a novel dissemination framework, eB4CAST, to document impact and effect of evidence-based research.

Evaluation: During the intervention study, physical assessments were measured for youth and surveys were completed by dyads. In dissemination, program outcomes were assessed. Conclusions and Implications: Based on 57\% retention, treatment versus control youth were higher for baseline adjusted BMI z-scores, fruit/vegetable intake (2.16 vs 1.67 cups/day; $\mathrm{P}=0.04)$ and dairy (0.57 cups/day; $\mathrm{P}=0.02)$. No intake differences remained after $1000 \mathrm{kcal}$ standardization. For treatment adults versus control, food resource management was 0.87 points higher $(\mathrm{P}=0.02)$. Program outcomes in treatment versus control was higher, for youth, cooking skills ( $\mathrm{P}=0.0002)$ and culinary self-efficacy $(\mathrm{P}=0.03)$ and, for adults: measure of "cooking, eating, playing together" $(\mathrm{P}=0.04)$. iCook $4-\mathrm{H}$ is being prepared for national distribution.

Funding: 2012-68001-19605

\section{NP35 WAVE $\sim$ Ripples for Change (Year 4 of 5): Baseline and Year 1 Exit Findings}

Siew SunWong, PhD, siewsun.wong@oregonstate.edu, Oregon State University, Ballard Hall 105E, OR 97331; Melinda Manore, PhD, RD, CSSD, FACSM, Oregon State University; Tonya Johnson, MPH; Christopher Scaffidi, PhD; Yu Meng, MS; Megan Patton-Lopez, DPH, RD; Mario Magaña Álvarez, MAIS; Cristian Curiel, BS

Objective: Prevent childhood obesity among high school athletes ages 14-19y in Oregon.

Description: Two year sport nutrition and life skills intervention with hands-on lessons in both physical and virtual worlds. In Fall 2015, 611 of 870 high school athletes from 13 schools in Oregon were enrolled in WAVE (intervention group $[\mathrm{IG}]=495$, comparison group $[\mathrm{CG}]=166$ ). Participants were $53.3 \%$ females, mean age of $15.3 \pm 1.2$ (13-18y), $57.6 \%$ participating in free or reduced lunch, 